Aim To investigate maternal, perinatal, and neonatal outcomes of pregnancies in women with type 1 diabetes in the University Maternity Hospital Limerick, Ireland.

Methods This retrospective descriptive study was conducted from charts identified from the diabetes in pregnancy register from $1^{\text {st }}$ July 2007 to $1^{\text {st }}$ July 2017 at University Maternity Hospital Limerick.

Results 17 women were identified from the register with juvenile onset ( $<18$ years) type 1 diabetes mellitus during the 10 year study period who gave birth to 23 live infants. Maternal diabetes related medical conditions included; diabetic retinopathy (29\%), hypertension (11.8\%), recurrent urinary tract infections $(11.8 \%)$, diabetic nephropathy (11.8\%), poor hypoglycaemia awareness (11.8\%), diabetic neuropathy $(5.9 \%)$ and cataracts $(5.9 \%) .11 .7 \%$ women had a past history of illicit drug use. $8.7 \%$ women were smoking at the time of booking. Antenatal obstetric complications included polyhydramnios $(17.4 \%)$, intrauterine growth restriction $(17.4 \%)$, pre-eclampsia (8.7\%), maternal hypoglycaemic seizure $(4.3 \%)$ and pregnancy induced hypertension (4.3\%). 39.1\% infants were born to primiparous women. $87 \%$ infants were bottle-fed. $73.9 \%$ births were unplanned pregnancies. Of $n=5$ infants born via vaginal delivery there were no cases of shoulder dystocia. $60.9 \%$ neonates were male. The mean gestation was 37.2 weeks (range 33.3 to 39.4 ). The mean birth weight was $3.57 \mathrm{~kg}$ (range 2.01 to 5.54). The median APGAR at 1 minute was 9 (range 5-9). The median APGAR at 5 minutes was 10 (range 4-10). $17.4 \%$ neonates required free flow $\mathrm{O} 2$ at birth and $8.7 \%$ required bag-mask ventilation. $95.7 \%$ neonates were admitted to the neonatal unit. Of the $n=23$ infants studied medical issues included; hypoglycaemia (60.9\%), jaundice requiring phototherapy $(43.5 \%)$, transient tachypnoea of the newborn (39.1\%), prematurity (30.4\%), macrosomia (17.4\%), low birth weight $(13 \%)$, poor feeding $(13 \%)$, polycythaemia $(8.7 \%)$, trisomy $21(4.3 \%)$, respiratory distress syndrome $(4.3 \%)$ and hyponatremia $(4.3 \%)$.

Conclusion Neonates born to mothers with juvenile onset T1DM should be considered a high risk group and clinicians should have a low threshold for admission to the neonatal unit.

\section{P294 CUSHING SYNDROME AND ADRENAL INSUFFICIENCY INDUCED BY HIGH DOSE PROLONGED INTRANASAL BETAMETHASONE}

${ }^{1}$ Claire Reynolds*, 'Pankaj Agrawal, ${ }^{1}$ Aisling McCann, ${ }^{2}$ Triona O'Sullivan, ${ }^{3}$ Muireann Ni Chroinin, 'Stephen MP O'Riordan. 'Department of Paediatric and Endocrinology, Cork University Hospital, Cork, Ireland; ${ }^{2}$ Pharmacy Department, Cork University Hospital, Cork, Ireland; ${ }^{3}$ Department of Paediatric and Respiratory Medicine, Cork University Hospital, Cork, Ireland

\subsection{6/archdischild-2019-epa.644}

Iatrogenic Cushing syndrome and secondary adrenal insufficiency due to intranasal steroids is rare in children. A 7 year old boy was referred to the paediatric endocrine clinic with suspected Cushing syndrome. He presented with excessive weight gain, marked striae and hypertension. His GP had performed a morning cortisol $<28 \mathrm{nmol} / \mathrm{L}(171-800 \mathrm{nmol} / \mathrm{L})$. He had a background history of obstructive sleep apnoea for which he had been prescribed Betnesol 0.1\% (Betamethasone sodium phosphate $1 \mathrm{mg} / \mathrm{ml}$ ) nasal drops and Montelukast 4 mg daily. Physical examination revealed hypertrichosis on his back, buffalo-humped neck, and purple striae on his legs and inner thighs. He was Tanner stage 1, normal genitalia. Auxology: weight $46.1 \mathrm{~kg}\left(>99.6^{\text {th }}\right.$ centile); height $124.6 \mathrm{~cm}\left(50^{\text {th }}\right.$ $75^{\text {th }}$ centile), body mass index (BMI) $30 \mathrm{~kg} / \mathrm{m} 2\left(>99.6^{\text {th }}\right.$ centile) and blood pressure was $124 / 72 \mathrm{mmHg}$ ( $>99^{\text {th }}$ centile). The drug history was reviewed in detail by our Paediatric Pharmacist and revealed that over the past 20 months, he had been using betamethasone nasal drops continuously, prescribed at a dose of 2 drops, twice daily, into each nostril. A total of 38 repeat prescriptions had been dispensed and he had been taking double the prescribed amount per month. The duration of use (20 months) also far exceeded the recommended indication (4-6weeks max). Laboratory investigations: blood glucose $4.7 \mathrm{mmol} / \mathrm{L}$, early morning cortisol $<28 \mathrm{mmol} / \mathrm{L}(171-$ $800 \mathrm{nmol} / \mathrm{L})$ and $\mathrm{ACTH}<0.2$ undetectable $(1.1-13.2 \mathrm{pmol} / \mathrm{L})$. Renin and aldosterone, anti-21 hydroxylase antibodies, thyroid function tests, IGF-1, coeliac screen, and renal/liver/bone were all normal. A short synacthen test (250micrograms) confirmed HPA axis suppression for our patient (cortisol concentrations: $<28 \mathrm{nmol} / \mathrm{L}$ at time: 0 mins, $30 \mathrm{mins}$ and $60 \mathrm{mins}$ ). This is gold standard diagnostic test when adrenal suppression is suspected. He was immediately commenced on replacement hydrocortisone therapy at a dose of $8-10 \mathrm{mg} / \mathrm{m}^{2} /$ day. He was also educated on emergency hydrocortisone administration and stress dosing. He will continue on hydrocortisone until his HPA axis recovers. Betamethsone is a potent steroid 25-30 times more potent than hydrocortisone

Conclusion We describe a case of iatrogenic Cushing syndrome and adrenal insufficiency secondary to administration of longterm high-dose betamethasone nasal drops. Very few cases to date have been reported in the literature in children. Cushing syndrome caused by intranasal steroids is a rare event and more commonly occurs after oral or topical steroid use. Our patient was taking double the recommended dose for more than 20 months. Paediatricians must be aware of the complications of nasal steroid administration and long-term use should be avoided

\section{P295 CHARACTERISTICS OF A POPULATION OF 311 PATIENTS OF BOTH SEXES WITH EARLY ONSET PUBERTAL SIGNS. DESCRIPTIVE STUDY}

${ }^{1}$ Paula Sol Ventura Wichner, 'Xavier Herrero, 'Maria Laura Llorca, 'Agustina Magistris, 'Zelmira Bosch, ${ }^{1}$ Marisa Torres Lacruz, ${ }^{2}$ Georges Denis Akel Perez ${ }^{*}$. ${ }^{1}$ Hospital de Nens, Barcelona, Spain; ${ }^{2}$ Paediatric Unit of Fundación Hospital de Nens de Barcelona, Barcelona, Spain

\subsection{6/archdischild-2019-epa.645}

Objective To assess anthropometric, biochemical and imaging studies (bone age, pelvic ultrasound and MRI) in patients who consulted the Endocrinology Department of the Hospital de Nens de Barcelona, referred by pediatricians to discard precocious puberty.

Methodology Retrospective descriptive study based on review of medical records, with first consultation between 2010 and 2018. Search from keyword in the diagnosis: puberty. The subjects were separated in 6 groups: involuted precocious breast development, early non-progressive breast development, central precocious puberty (PPC), peripheral precocious 
puberty (PPP), advanced puberty and rapidly progressive puberty. Exclusion criteria: not going to follow-up visits. Statistical analysis by SPSS 23 .

Results A total of 311 subjects (14 men, 297 women) who were referred by their pediatricians, attended the first visit to the endocrinology department with an average age of 7.8 years (95\% CI: 7.7-8). The distribution of subjects was: involuted precocious breast development $(n=25)$, non-progressive precocious breast development $(\mathrm{n}=19)$, PPC $(\mathrm{n}=156)$, PPP $(\mathrm{n}=3)$, advanced puberty $(\mathrm{n}=88)$ and rapidly progressive puberty $(n=20)$. Male subjects who were diagnosed of precocious puberty had at first visit a mean age of 8.7 years (95\% CI: 7.8-9.5), and women had a mean age of 7.7 years (95\% CI: 7.5-8). There are significant differences regarding bone age at diagnosis (more advanced in central precocious puberty and rapidly progressive puberty). There is a statistically significant association between precocious puberty and the fact of being adopted (X2: 11.262; p: 0.046). The LH at 3 hours of the GnRH test was significantly higher in the PPC group (mean value of LH 14UI/L, IC95\%: 11.5-16.6) compared to the others. Patients were identified who did not meet the classic criteria of the diagnosis of PPC in the Procrin test, however they presented clinical characteristics in the follow-up that led to the diagnosis. MRI: significant association between having MRI findings and presenting PPC (X2: 38.262; p: 0. 000), however incidental findings where found in MRI of patients with advanced puberty. A total of $69 \%$ of patients with PPC and $17.6 \%$ patients with advanced puberty (including here the rapidly progressive forms) received treatment.

Conclusions We present the data of a population of children of both sexes with clinical manifestations suggestive of precocious/advanced puberty evaluated and followed between the years 2010 and 2018. The results coincide with those described in previous studies.

\section{P296 GENU VALGUM AS A RARE CLINICAL MANIFESTATION IN CHILD WITH PRIMARY HYPERPARATHYROIDISM: A CASE REPORT}

Yaulia Yanrismet*, Bambang Tridjaja. Departement of Child Health, Faculty of Medicine University of Indonesia, Jakarta, Indonesia

\subsection{6/archdischild-2019-epa.646}

Background Primary hyperparathyroidism (PHPT) is a rare case in children with the incidence of $2-5: 100,000$. Its manifestations vary greatly from asymptomatic to severe complications. Genu valgum is a scarce clinical manifestation of PHPT in children and often misdiagnosed with rickets. There were scanty case reports about genu valgum in children with PHPT worldwide. It is generally sporadic and nearly $65 \%$ caused by adenomas; curable by surgical therapy. Delayed in diagnosis increases the morbidity and mortality rate

Objectives To report a rare case and its scarce clinical manifestation in children

Case presentation A 13-year-old boy with bilateral genu valgum was referred with rickets. He also had other clinical feature of skeletal abnormalities such as progressive difficulty in walking, bone and joint pain and muscle weakness since six months prior to admission. He was normal at birth. Formerly he was diagnosed as rickets and had calcium and vitamin D therapy, but no improvement. He, thereafter, referred to the National referral hospital CiptoMangunkusumo hospital. Skeletal survey revealed decreased bone density by epiphyseal plate widening, bilateral lateral clavicle tip, sacroiliac joint bilateral, and symphysis pubic resorption with rugger-jersey spine, slipped capital femoral epiphysis bilateral. Metabolic profile showed low vitamin D level with hypercalcemia and mild hypophosphatemia. Intact parathyroid level was very high (1301 pg/ml). Bone mineral density below the value of children in his age (whole body z-score was -5,5 and AP spine zscore was -4,5). Kidney function test and liver function test were normal. MSCT of the larynx showed solid lesions with the contrast in the right thyroid gland inferoposterior pole as high as vertebrae cervical 6 to thoracic 1, confirmed with dual time Tc99 parathyroid scintigraphy with the appearance of pathological activity at the inferior of the right thyroid suspected as parathyroid adenoma. He underwent parathyroidectomy surgery; the results of anatomical pathology examination in accordance with adenomas. Post surgery he experienced hungry bone syndrome.

Conclusion Genu valgum is a scarce clinical manifestation in children with PHPT which mostly wrongly diagnosed and treated as rickets. This condition occurs in PHPT children at pubertal aged with unknown mechanism. The misstep of making the diagnosis will lead to higher morbidity and mortality. Therefore, early diagnosis in children with genu valgum who suffer PHPT would prevent further bone damage and irreversible complications. A panel of test which includes parathyroid serum examination is warranted in metabolic bone disease.

Genu valgum, primary hyperparathyroidism, case report

\section{P297 CONSTANT UNCERTAINTY: MOTHERS' EXPERIENCES OF CARING FOR CHILDREN RECEIVING GROWTH HORMONE TREATMENT (GHT)}

${ }^{1,2}$ Rasha Alsaigh*, ${ }^{2}$ Imelda Coyne. ${ }^{1}$ King Abdulaziz University, College of Nursing, Jeddah, Saudi Arabia; ${ }^{2}$ Trinity College Dublin, School of Nursing and Midwifery, Dublin , Ireland

\subsection{6/archdischild-2019-epa.647}

Uncertainty has been noted as a significant feature of parental experiences of childhood chronic illnesses needing medical treatments in general and growth hormone treatment (GHT) in particular. However, there is insufficient research exploring the extent of which uncertainty features in the experiences of parents caring for children receiving GHT or the dimensions, which their uncertainty may take. Drawn from 16 in depth interviews and eight diaries from 16 mothers from the Republic of Ireland who had children with a growth disorder requiring daily administration of GHT, this paper highlights their experiences of uncertainty when it comes to dealing with GHT. It was concluded that the dimensions of uncertainty experienced by the mothers were: diagnostic, treatment, future and social stigma uncertainty. It also demonstrates the significant challenges of coping with these uncertainties. By recognising the dimensions of uncertainty faced by mothers caring for children receiving GHT, health care professionals can anticipate these uncertainties and help minimise some of the stress and anxiety associated with them. 